\title{
Computer genomics of regulatory single nucleotide polymorphisms in neurodegenerative diseases based on metabolic pathways models
}

\author{
P. Shanmughavel ${ }^{1 *}$, R. Sathishkumar ${ }^{1}$, M.P. Ponomarenko ${ }^{2,3}$, M.R. Khantemirova ${ }^{3}$, \\ L.E. Tabikhanova ${ }^{3}$, Y.L. Orlov ${ }^{2,3}$ \\ ${ }^{1}$ Department of Bioinformatics, Bharathiar University, Coimbatore, Tamilnadu, India \\ ${ }^{2}$ Novosibirsk State University, Novosibirsk, Russia \\ ${ }^{3}$ Institute of Cytology and Genetics SB RAS, Novosibirsk, Russia \\ *e-mail:shanmughavel@buc.edu.in
}

Key words: non-coding RNA, transcriptomics, stress response, crop plants

Motivation and Aim: Neurodegenerative diseases present challenging problem for growing population in Asian countries demanding joint research efforts based on genetics data and bioinformatics tools. Our work will focus on integration of national sources and computer tools developed for regulatory single nucleotide polymorphisms (SNP) analysis [1]. Object of the study is neurodegenerative diseases in context of regulatory effects of SNPs. SNPs play important role in neurodegenerative diseases such as Parkinson's disease, Alzheimer's disease, Huntington's disease, Amyotrophic lateral sclerosis (ALS), Motor neuron disease, Schizophrenia.

Methods and Algorithms: The modeling approaches is based on integration of data, analysis of sequence contexts, estimation of regulatory effects of single nucleotide polymorphisms in metabolic pathways and gene networks. We develop novel software for supercomputer analysis of omics data, regulatory SNP prediction, integrated databases on nucleotide polymorphisms in neurodegenerative diseases. Aim is computational prognostic effects of polymorphysms and treatment of neurodegenerative diseases based on personalized genome data. We will use several tools for SNP prediction. The tolerated and deleterious SNPs will be predicted using SIFT (Sorting Intolerant from Tolerant (SIFT, version 2). For regulatory SNP (rSNP) effects we use tool SNP_TATA_comparator developed at the Institute of Cytology and Genetics SB RAS, Novosibirsk [2].

Results: We analyze the SNP responsible for these neurodegenerative diseases and compiled available data and computer tools to a web-resource.

Conclusion: The modeling approaches will be based on integration of genomics data, analysis of sequence contexts, estimation of regulatory effects of single nucleotide polymorphisms in metabolic pathways and gene networks. We continue work on integration of stress-response data and gene sets using available data sources.

Acknowledgements: The research has been supported by RFBR and Indian DST. Computing done at Siberian Supercomputer center SB RAS was supported by budget project 0324-2018-0017.

\section{References}

1. Sachidanandam R. et al. (2001) A map of human genome sequence variation containing 1.42 million single nucleotide polymorphisms. Nature. 409:928-933.

2. Ponomarenko M. et al. (2015) How to use SNP_TATA_Comparator to find a significant change in gene expression caused by the regulatory SNP of this gene's promoter via a change in affinity of the TATAbinding protein for this promoter. Biomed Res. Int. 2015:359835. 